\title{
基于人机互动的数字化城市设计 城市设计第四代范型刍议
}

\section{Digital Urban Design Based on Human-Computer Interaction: Discussion on the Fourth Generation of Urban Design}

王建国

Wang Jianguo

摘要: 在城市设计发展历程中, 其依托的理论和技术方 法一直与时俱进。城市设计先后走过了四代范型, 亦即: 建筑学基本原理主导、以城市三维形体组织为对象的第 一代传统城市设计, 以科技支撑、功能区划、三维空间 抽象组织为特征的第二代现代主义城市设计, 基于 “生 态优先和环境可持续性原则的第三代绿色城市设计, 和 今天正逐渐浮现的基于人机互动的第四代数字化城市设 计。第四代城市设计以形态整体性理论重构为目标, 并 以人机互动的数字技术方法工具变革为核心特征。通过 众多案例的科学研究实践成果表明, 在可见的未来, 规 划设计编制将有望实现“从数字采集到数字设计, 再到 数字管理”的跨越。

Abstract: The theories and technique methods relied on by urban design always keep pace with the times in its development. Urban design has gone through four generations of paradigms, which are: The first generation of traditional urban design dominated by the basic principles of architecture, the second generation modernist urban design characterized by scientific and technological support, functional zoning and 3D space abstract organization, the third generation of green urban design based on the "ecofirst' principle and the environmental sustainability, and the fourth generation of digital city design which gradually emerging based on human-computer interaction. The fourth generation of urban design aims at the reconstruction of the modal holism theory and takes the transformation of the human-computer interaction's digital technology method as the core feature. The scientific research and practical results of many cases show that in the foreseeable future planning and design will be expected to achieve 'from digital collection to digital design, from digital design to digital management'.

关键词: 城市设计; 范型; 数字化

Keywords: Urban Design; Paradigm; Digitalization

作者: 王建国, 东南大学建筑学院教授, 博导; 中国工程 院院士

\section{1 城市设计范型的发展历程}

城市设计, 古已有之。人类自希腊、古罗马、中国等早期文明的城市 营造活动中, 形成了朴素的城市空间设计经验, 并在欧洲、阿拉伯地区、亚 洲等不同文化体系中发扬光大，形成了城市设计的萌芽。千百年以来，在 城市设计的发展历程中, 其关注城市空间形态建构和组织的基本概念并没 有发生根本性的改变, 但是城市设计依托的理论和技术方法一直与时俱进。

（1）第一代传统城市设计。1920 年以前, 城市设计所遵循的价值取 向和方法论系统基本上以建筑学基本原理和古典美学为指导（图 1), 对 较大空间范围内的建筑进行三维形体控制, 直觉感性多于科学理性, 这也 是该阶段城市设计范型的基本特点。代表人物如西特、沙里宁等, 其后吉 伯德、卡伦、芦原义信等又很好地继承和发展了这一理论。

（2）第二代现代主义城市设计。工业革命之后, 城市空间环境和物质 形态发生了深刻变化。城市人口与用地规模急剧膨胀, 超出了常规建筑手 段所能驾驭的能力, 人们逐渐意识到只有通过整体的形态规划才能摆脱城 镇发展的困境, 产生现代主义城市设计。该阶段城市设计者不再仅仅关注 于城市空间的艺术处理和美学效果, 而是遵循经济和技术的理性准侧, 以

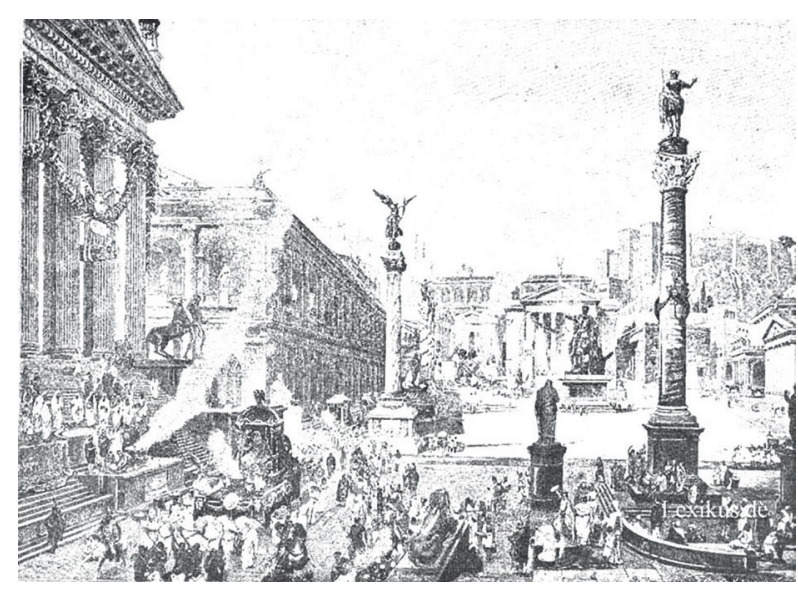

图 1 西特《遵循艺术原则的城市设计》插图 资料来源 : 参考文献 [1] 
科技支撑、技术美学、功能区划、三维空间抽象组织为特征, 建构城市物质环境的总体理论与方案, 以寻求一系列城市问 题的解决方案, 代表人物如柯布西耶 (图 2)、培根 (Bacon)、 克里尔兄弟等。在二次大战后的城市建设中, 现代主义城市 设计的内在目标、方法论又由于世界性社会发展的新特点而 产生了新的发展, 后期发展的主要代表人物包括林奇、雅各 布斯、“小组 $10 ”$ 、拉波波特、罗西及柯林 - 罗和弗瑞德 - 科 特等。

（3）第三代绿色城市设计。1970 年代以来, 现代主义思 潮退去, 规划学者发现纯粹的空间形态设计并不能一劳永逸地 解决城市问题, 进而反对现代主义的机器美学, 肯定城市规划 的复杂性与矛盾性。城市设计在对象范围、工作内容、设计 方法乃至指导思想上也有了进一步的发展, 其中最重要的是 对城市生态与环境问题的认识反思和觉醒, 并努力将这种认 识反映到城市设计实践中, 即“绿色城市设计”。该阶段城市 设计通过把握和运用以往城市建设所忽视的自然生态的特点 和规律, 贯彻整体优先和生态优先准侧, 力图创造一个人工 环境与自然环境和谐共存、面向可持续发展的理想城市环境。 为此, 规划师充分运用各种可能的科学技术, 特别是城市生态 学和景观建筑学的一些方法来实现这一目标, 代表人物为麦克 哈格、西蒙兹、霍夫等。与以往相比, 绿色城市设计更加注重 城市建设内在的质量, 而非外显的数量, 强调提高城市的 “适 居性” 和人的生活环境质量, 从而改善城市整体空间环境与景 观, 促进城市环境建设的可持续发展。此外巴奈特、希尔瓦尼、 特兰西克、斯滕伯格等在如何将城市设计融人城市公共政策, 以及形成整体性的现代城市设计方法等方面进行了积极有益 的探索, 对城市设计范型的进一步完善具有重要作用。

至此, 可以从城市设计的发展史中总结出三代范型, 亦 即：建筑学基本原理主导、以城市三维形体组织为对象的第 一代传统城市设计, 以科技支撑、功能区划、三维空间抽象 组织为特征的第二代现代主义城市设计, 以及基于 “生态优

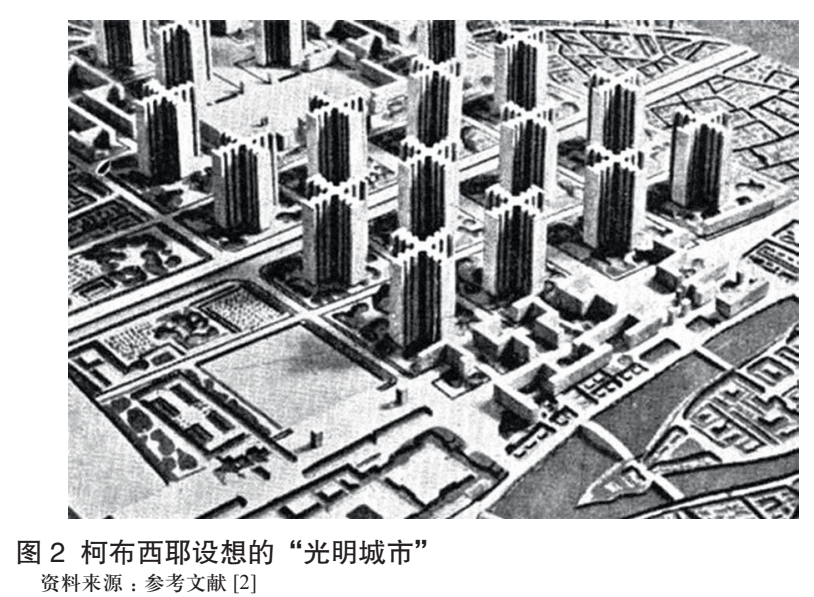

先和环境可持续性” 原则的第三代绿色城市设计。而本文将 着重讨论的, 则是当下正逐渐浮现的不同于以往的城市设计 新范型一一基于人机互动的第四代数字化城市设计。

\section{2 第四代城市设计出现的时代背景}

\section{1 数字技术进步为城市设计整体性建构提供了可能}

城市设计历史上曾经受到量子力学、生物学、神经学和 胚胎学等影响, 出现了对于整体性的关注。在此基础上, 美 国加州大学伯克利分校的亚历山大于 1987 年出版了《城市 设计新方法》 ${ }^{[4]}$ 。该书记录了亚历山大团队 1978 年以来关于 城市设计的研究实验, 试图重新诠释城镇形态的整体生长, 提出城镇在其特有的整体性法则下发展, 而这种整体性法则 针对的主要是 “有机的复杂性”, 这种方法可被描述为“生 成性的”。林奇同样一生都在城市规划和设计领域建构城市 整体性; 在更加广义的范围内, 道克希亚迪斯的人居环境理 论 (Ekistics)、沙里宁的 “有机疏散” 理论等都关注城市形 态的整体性意义。从定量分析的角度看, 亚历山大作为数学 和计算机方面的学者, 曾经在更早些时候尝试用一个整合系 统来设计印度的村庄 ${ }^{[5]}$, 但是并没有成功, 因为人居环境设 计问题十分复杂, 不能任意简化。后来, 亚历山大用模式语 言 (pattern language) 的菜单方式论述城市规划和设计的原 理, 实际上放弃了总体性的解题路径。其主要原因是当时的 探索受到了计算机技术工具发展的瓶颈限制。对于此问题, 笔者也有同样的经历和体会一2003 年在主持南京老城高 度控制引导研究时, 尝试利用数字技术建构城市用地的影响 因子互动模型, 并得到当时规划局的首肯, 然而受当时计算 能力的局限, 只能通过简化要素和减少城市用地地块划分的 数量来操作信息集取和设计处理过程。近年来, 数字技术发 展的突飞猛进为我们重新建构城市形态及其设计的整体性提 供了可能。对于同样的命题, 在 2015 年重新研究时, 地块 数量就比 2003 年增加了约 7 倍（2003 年 756 个, 2015 年 5500 个), 成果的精度和实用性远非先前可比。

\section{2 城市规划和城市设计编制实施的实效性亟待提升}

整体多重尺度上空间形态的发展引导、控制管理和科学 规划设计是当代中国城镇建设普遍面临的新课题。当下人们 普遍诟病的 “千城一面” 和 “万楼一貌”, 以及 “奇奇怪怪” (此处泛指城市建设中形态不协调现象), 很大程度上是由 “自上而下”和 “自下而上”两方面原因及其相互矛盾所造 成的, 即现行的法定城市规划编制和管理体系内的缺位, 控 规实施中依据经验套路对较大尺度城市区块的 “无差别化” 的指标设定和管理; 同时一个时期城市发展的经济优先思路 则导致了规划权威性遭受挑战并一再向资本和市场让步, 致 
使城市形态整体失控。

政府一向把城市规划作为城市发展和建设的龙头, 我国 大中城市一般都有上级政府批准的城市总体规划, 这些规划 无疑已经成为政府制定发展政策、组织城市建设的重要依据。 控制性详细规划虽然在城市各类用地安排和确定建筑设计要 点方面具有技术支撑的作用, 但是, 对于什么是人们在生活 活动和感知层面觉得 “好的、协调有序的” 的城市空间形态, 以及城市品质中包含的 “文化理性”，如城市的社会文化、 历史发展、艺术特色等, 始终没有找到科学合理的、可具体 操作的, 同时又具有普适意义的研究切人途径和技术方法, 函待城市设计予以补缺。

过去的以三维形态效果图和终极蓝图为表现形式的城市 设计引发了一定的批评, 很多人抱怨城市规划是 “纸上画画、 墙上挂挂”。究其原因, 业界已经有很多论述, 主要还是两 个方面的问题没能有效解决：一是规划或者设计编制的科学 性和包容性; 二是未能很好揭示和把握城市演进发展的特征 和规律, 导致规划和设计无法有效纳人城市发展的政策架构 和管理体系，存在 “一管就死，一放就乱”的现象。“一管 就死” 说明规划编制的专业内容与实际的社会需求和市场活 力要求之间存在 “水土不服”, 不能同向发力。“一放就乱” 说明规划编制的基本原则缺乏科学支撑，专业底线不清，导 致管理缺乏抓手且人为裁量空间过大。回顾历史, 这样的问 题其实在发达国家也是反复讨论的焦点, 基于刚性量化指标 的形态管控与本质上是新陈代谢的城市形态有序成长始终存 在内在的深刻矛盾。

所以，城市规划和城市设计编制及其实施的实效性是一 个迫切需要破解的关键科学问题。城市环境的广延性、城市 建设的连续性、管理体制的多样性、具体决策的分散性 ${ }^{[6]}$, 决定了城市设计绝不仅仅是专业技术问题，而是应该让专业 技术及成果形成学理坚实、底线明唽、包容开放并可持续修 正优化的实操依据。时代呼唤一种内外兼修, 兼顾城市空间 形态 “高度、宽度、深度、精度 (细节)、温度 (人性场所)” 的新一代城市设计, 这就是我们所构想提出的第四代基于人 机互动的数字化城市设计范型。

\section{3 第四代城市设计的特征总结}

\section{1 全尺度的设计对象, 贯彻城市形态整体性思考}

中国城市设计所要应对的对象范围主要涉及范围以平方 公里计量的城市形态（图 3), 其社会需求和数量远远大于中 小尺度的、以物质形态建设为特点的局部城市设计。大尺度 城市设计所涉范围大致类比成中国法定城市规划体系中的控
规编制单元及以上尺度的规划编制单元。通常, 对于局部的 形态变化和引导控制, 专业人员可以根据经验和建筑学知识 予以驾驭, 这也是基于视觉美学原则的传统城市设计的主要 工作内容。但当下中国城市设计常常依托法定规划编制的规 模尺度, 动轧涉及数平方公里乃至数十平方公里的城市地区, 已不是用常规的城市设计概念、原则和技术方法即可轻易掌 控, 经典城市设计原理对此已然失效。30多年来的实践证明, 仅仅靠城市规划塑造一座城市的良好空间形态（尤其是城市 的特色风貌), 以及营造富有活力的场所环境是远远不够的。 近年一些城市开始在控制性详细规划中新增特色意图区和城 市设计内容, 城市规划和城市设计的结构性关联有所加强, 但究其本质, 城市的优美形态、城市活力和场所特点主要是 通过设计营造出来的。现在有学者开始提 “用设计做规划” 就是为了弥补以往规划在特色塑造方面的欠缺 ${ }^{1}$ 。

什么是大尺度城市设计? 按照图纸表达的朴素认识, 建 筑设计一般是 1:10 (建筑节点大样) 1:100 (建筑基本图纸) 到 1:500 1:1000 (总图), 建筑群带场地等小尺度的城市设 计其一般图纸比例在 1:500 1:2000 之间, 中等尺度的城市 设计图纸表达一般在 1:1 000 1:5 000, 大尺度城市设计主要 指片区规模, 图纸一般在 1:5 000 1:20 000。从图纸表达能 力看, 能够出现具体三维建筑物理形态一般为 1:200 1:1 000 (前者表达建筑设计意图和部分细节, 后者主要表达建筑与 环境的关系), 三维立体形态极限应该在 1:5000 (100 m 高 楼相当于 $2 \mathrm{~cm}$ )。从城市设计一般概念上, $1: 5000$ 以上基本 与城市规划更加相关（也是国土资源部门用于沿海发达地区 的用地管理尺度)，一般超出了真实的人的空间认知和感知 能力。以往的城市设计大多通过空间结构表达、重点空间形

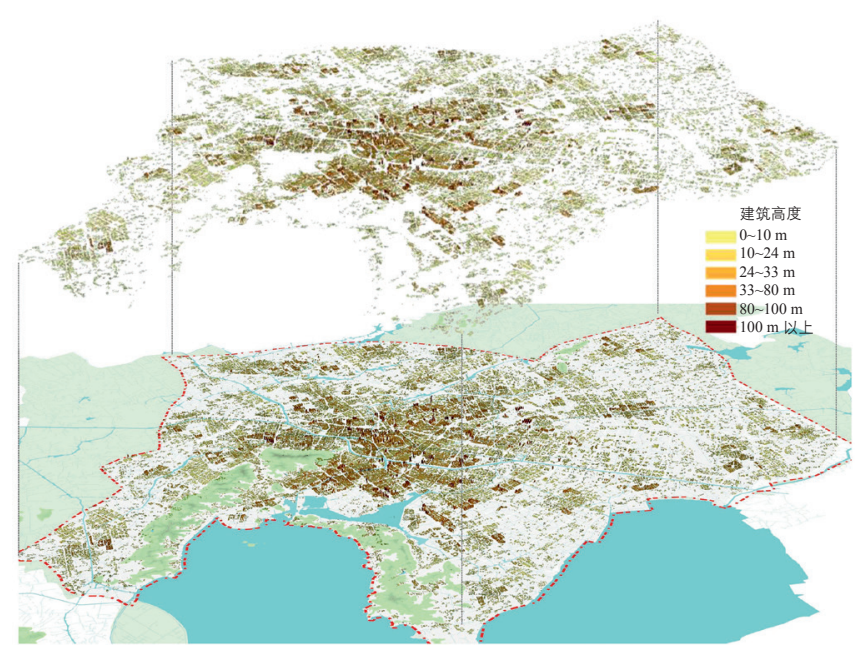

图 3 中国城市设计面临的大尺度城市空间形态问题 资料来源 : 参考文献 [7]

(1) 参见朱子瑜. 用设计做规划 [R]. 中国城市规划年会 “规划作用与规划改革”, 2014。 
态强化和总体加上局部放大的图示表达大尺度城市设计（如 培根书里所表达的那样)。但是真实的全尺度形态关联要素 的认知和把握并没有得到解决, 特别是当城市设计的对象达 到全局空间形态的尺度, 就必须用新的理论分析方法和技术 辨析手段揭示城市空间形态要素系统、建构机理乃至演化规 律。在操作方式上, 大尺度城市设计一般需要不同相关领域 的专业人员 “跨界” 集群组织, 特别是与各种专业性城市规 划编制人员和精通数字运算的专家分工协作完成。近年来, 大量总体城市设计的设计范围在数百平方公里以上, 已经远 远超出传统城市设计的操作范畴, 而在这些项目实践中, 全 尺度的设计范畴使得对于城市空间的整体性思考得到很好的 贯彻（图 4)。

\section{2 数字化的设计方法, 带来革新性的城市空间认知与 思考方式}

数字化实体虚拟是市民与城市建设决策者参与规划设计 并与专业人员沟通的常用方式。1980 年代, 早期的城市空间
数字化是通过 $C A D$ 、电子图学 (CG) 和 $V R$ 、卫星影像、照 相测量、GIS 等实现的, 正是这些技术使得城市景观的视觉数 字化与城市信息的整合成为可能。国内外不少城市的规划管理 部门已经有了不同精度的三维立体的城市空间信息数据库, 为 城市建设提供了很好的帮助。在柏林、格拉斯哥、赫尔辛基、 洛杉矶等城市, 已经在此基础上初步实现了寻路、导览和虚拟 旅游等互动式的数字城市功能。谷歌地图中的 Panoramio, 可 以通过人们上传的海量照片, 使电脑前的用户拥有身临其境的 视觉感受。建构三维城市模型的常用方式包括 : 传统的计算机 建筑辅助设计的建模、数字化照相测量技术和地理信息系统。

越来越多的证据表明, 数字技术的日新月异已经使城市 设计技术创新成为可能。数字技术深刻改变了我们看待世界 物质形态和社会构架的认知和看法, 某种意义上是一种全新 的世界认知、知识体系和方法建构。例如, 基于夜间灯光亮 度和密度分布所看到的世界城市化整体图景（比数字表述更 加整体直观）、基于交通时间可达性而形成的世界地图（不 再是以往仅仅以物理空间及其城市之间的距离为依据), 以

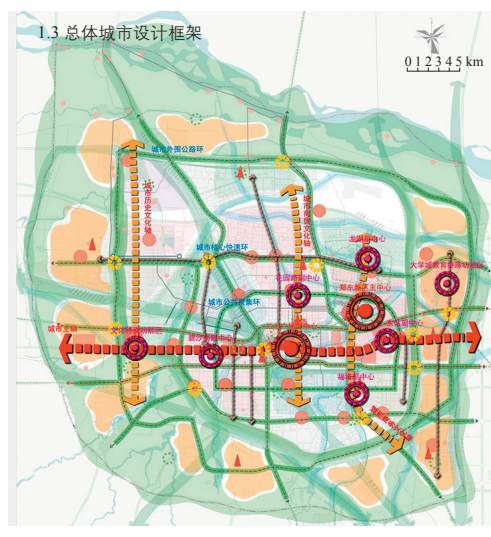

总体层面设计

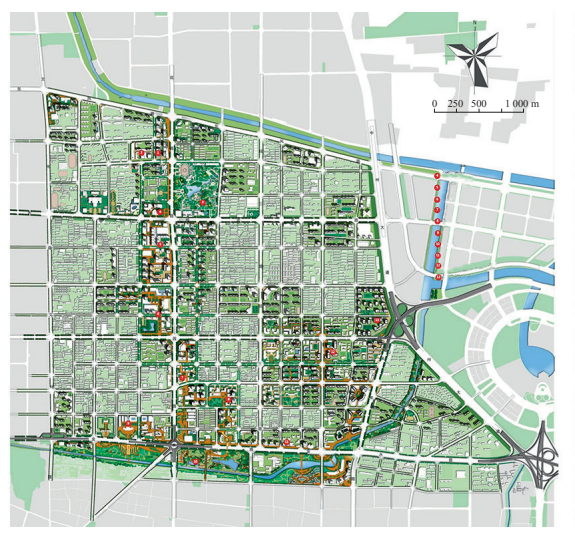

片区层面设计

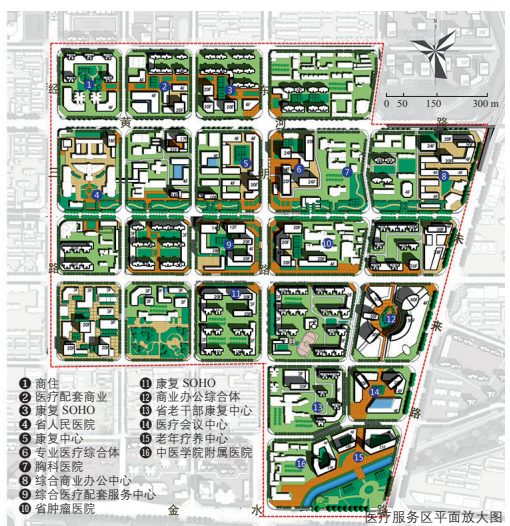

地段层面设计

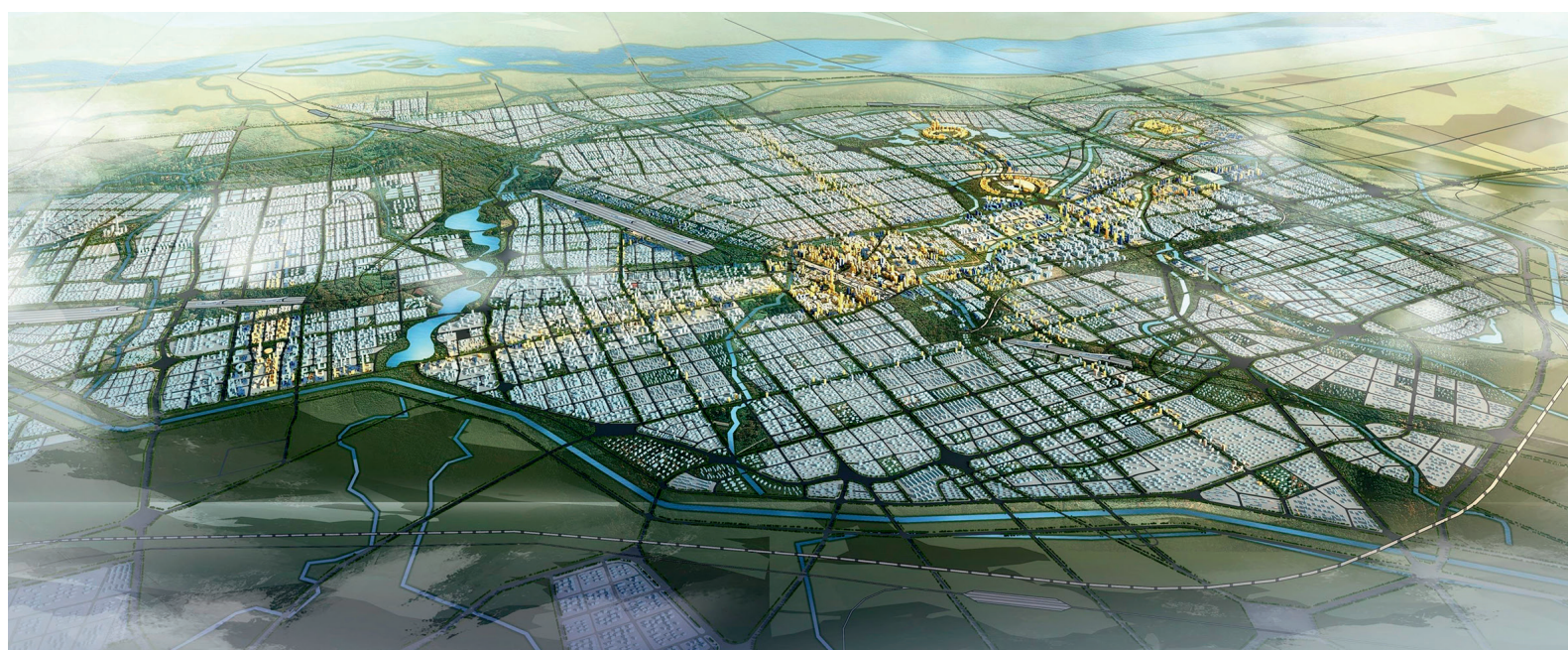

城市设计空间效果

图 4 郑州中心城区总体城市设计, 设计范围达到数百平方公里 资料来源 : 参考文献 $[8]$ 
及根据相关历史信息绘制的数字化历史全息地图（将城市历 史信息可视化并进行空间关联）等。

以往的问卷、访谈方式虽然具有面对面、个体针对性、 详略可控的优点, 但实施操作成本很大。大数据技术的出现部 分改变了传统的公众参与和调研方式, 例如今天可以基于百度 数据公众偏好度 (兴趣点 [POI: Point of Interest]) 和谷歌地图, 由游客、居民、专业人员或者政府机构参与完成 Panoramio 城市意象采集, 汇总形成城市设计所需的基于 POI 的数字地 图（图 5) 和城市意象偏好度信息 ${ }^{1}$ 。这些信息不仅具有实时 性的优点, 而且可以不断增加数据量, 完善优化数据精度, 方 便即时性变更, 这都是以往无法实现的。在城市设计调研中, 先通过大数据方法进行居民的海量信息获取, 然后在此基础上 进行针对性的、分门别类的问卷和访谈就会事半功倍。

在地性的现场调研也是如此, 受制于城市设计编制的专 业团队的规模和时间成本，调研工作总难免有所疏漏。而基 础信息掌握的充分性、整体性和有效性的不足，则导致城市 设计编制成果领导不满意、专家不认同、群众不接受。如果
有了今天的 Panoramio 等工具对人们城市景观意象偏好性进 行的海量信息采集, 百度地图和高德地图提供的实时交通和 基于 POI 的城市公共设施信息（学校、餐馆、超市、加油站 等), 以及无人机延时摄影、重要场地连续摄像等城镇空间 环境和人群活动集聚度的数据采集方法, 就可以在海量信息 整体采集和实地调研之间做到有机结合, 把控好这二者关系 的度，更加有效地完成对关键现场信息的调研。

在具体的设计层面, 通过共享性的城市规划数据集取、 分析和管理平台, 城市设计将不会再由于三维形态评判因人 而异的主观性而无法应对大尺度城市空间形态。在一定的场 合, 数字化城市设计可以相对独立于规划而自成逻辑系统, 并获得问题解决的独特路径。大数据加深并改变了人们对城 市形态和空间组织规律性的认识, 一定程度上重构了人们心 目中对城市形态的认知图式, 其数据库已经成为城市设计全 新的成果形式, 而且可以直接植人当下以信息电子化为特征 的规划管理。数据库成果同时可以通过整体关联联动的方式, 实现持续完善的动态更新。

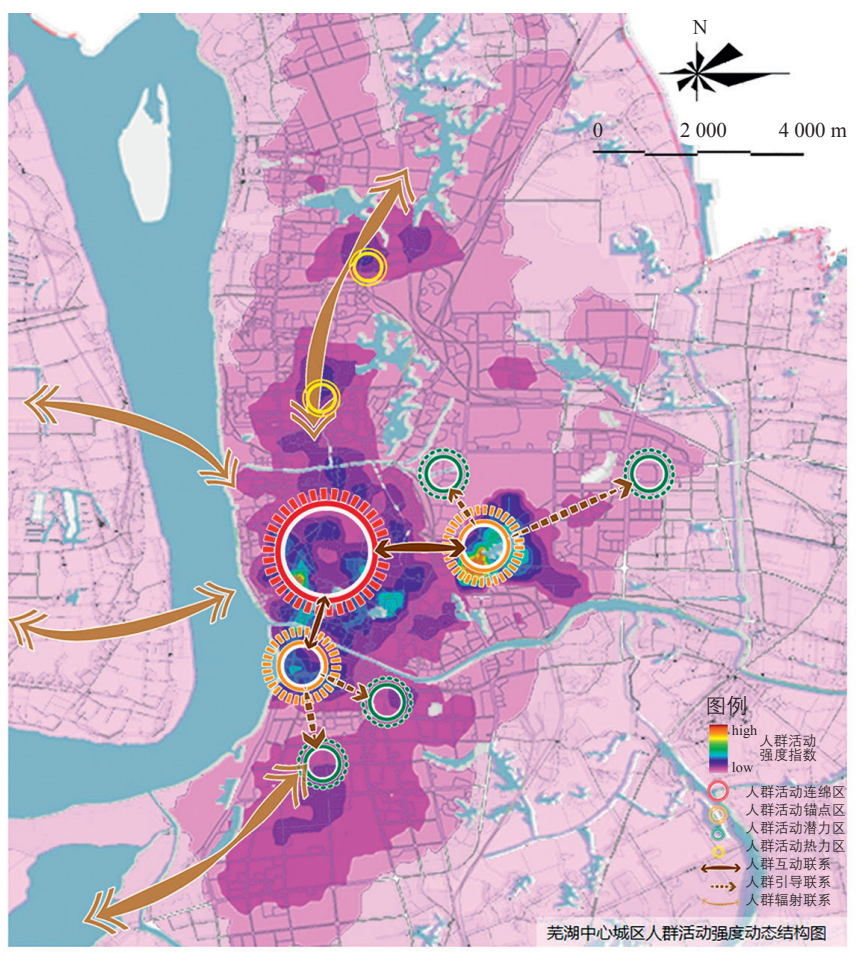

基于手机信令数据的人群活动分析

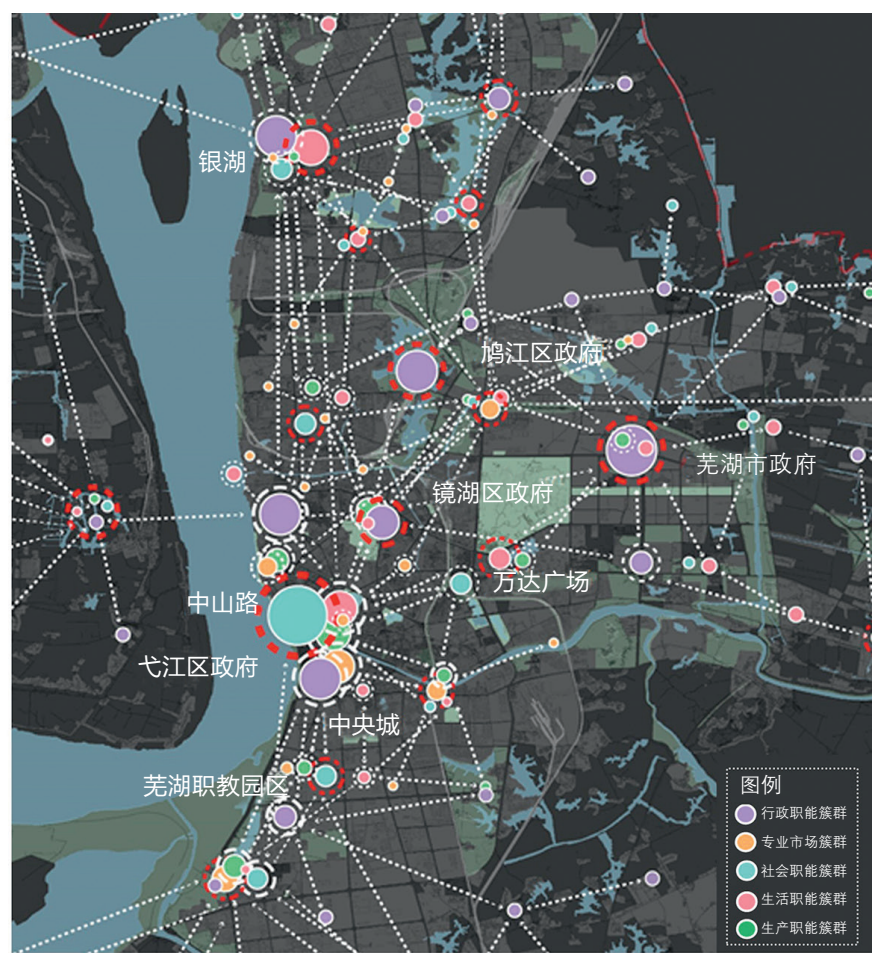

基于 POI 数据的产业业态分析

\section{图 5 基于大数据的城市规划分析}

资料来源 : 参考文献 [10]

(1) Google Earth 是一款由谷歌公司开发的虚拟地球仪软件, 它集合了卫星图片、航空照相、一般性的地图信息等海量信息, 通过这个载体, 数 以千万计的用户可以将信息上传, 这些信息将被审核、篎选, 最终以图层形式公布在 Google Earth 上面。其中 Panoramio 就是一个图层, 数 千万的用户, 包括城市居民、游客, 以及城市、建筑专业的个人、机构、政府组织等等, 可以将他们在地球上任何一个地方拍的照片上传到 Panoramio。谷歌将审核这些照片的真实性和清晰度, 确认后这些照片将被发布, 并且在照片拍摄地作出标记。一个地方作出的照片标记越多, 也就意味这个地方的受关注程度越高。 


\section{3 人机互动的设计过程, 提升规划成果的实施性}

海森堡的“测不准原理” 对于因果律和决定率的怀疑 ${ }^{[11]}$, 在城市研究领域产生了广泛的影响。城市设计编制成果是否 能够产生预期的实施效果, 需要长期的验证与完善。此前城 市设计导则因为缺乏与时俱进的修正完善机制, 真正融人真 实的城市规划建设长效管理仍有相当缺陷。美国城市设计学 者雪瓦尼 (Shirvani) ${ }^{[12]}$ 和培根 ${ }^{[13]}$ 提出的 “导则” (guideline) 和“维护” (maintenance) 两项城市设计成果形式虽与环境 长效管理有关, 但主要是从设计原理的角度定性提出, 并非 基于系统整体的多因子、大样本海量信息而得出。《现代城 市设计理论和方法》一书曾经在方法论一章中写到第二代设 计方法, 初步设想了基于设计者和用户无知对称、相互学习、 试错探寻和反馈机制的方法 ${ }^{[6]}$ 。

对于城市大尺度空间形态而言, 数字化城市设计是一种 能真正付诸实施的城市设计, 它不仅包含了相对完整、系统 和可靠的多源相关信息的集取、分析、综合、集成, 而且包 含了面对设计实施、运维管理、城市可持续发展和必要弹性 的物质空间形态基础性建构, 可看作最终形式的雏形。人机 互动的数字化城市设计具有包容发展变化和持续优化纠错的 属性, 通过设置一定值域 “容错” 进而实现系统的正常运转。 同时, 数字化成果明确了城市形态健康生成和成长的城市设 计把控底限和有限选择, 摒弃了主观定性决策的信息依据的 不完备性和无边际的、因人而异的选择空间。

\section{4 第四代城市设计的展望}

从目前的实践情况看, 数字化城市设计既可归属于上位 高层次规划, 也可与规划合体作用, 其基于科学量化的成果 特点, 使得 “向权力讲授真理” 有了真正比较靠谱的依据。 未来通过掌握大数据技术工具, 全局整体地把握各种与空间、 资源、人的活动等方面的信息及其与城市规划和设计相关的 意义, 就可能更好地实现城市设计初衷所强调的公平和效率 原则。虽然科学并不能完全取代人们体验环境的感受及绿色 理念的贯彻, 但在此基础上与前述范型设计内容彼此校核和 融合, 城市设计成果的科学性就提升了关键的一步。

当然, 从公众和普通市民感受、使用、品评和分享的 角度看, 城市设计毕竟是要处理综合性规划设计专业问题并 在此基础上做出创新性设计, 城市设计实施的成果优劣需要 接受社会和公众的评价。所以, 信息采集、归类分析和数据 库建立只是城市设计的第一步工作, 或者说是解决了基于数 字化的共性科学问题, 奠定了比以往更为客观理性的设计参 照依据和决策基础。数字化城市设计具有可量化定格、过程 开放、允许实时修改且整体联动的特点, 它还必须与主要依 据设计创意和设计者因人而异的随机性城市设计过程结合起
来。最终, 规划设计编制过程实现 “从数字采集到数字设计, 再从数字设计到数字管理” 的跨越, 城市设计就真正可以实 现 “第四代范型”。

这一范型已经在国内一些城市设计编制案例中得到一定 程度的实验和验证, 包括南京老城高度形态研究、常州老城 容积率及单元地块建筑布局的城市设计研究、西湖东岸城市 景观规划、郑州市中心城区总体城市设计等。同济大学王德 团队, 东南大学杨俊宴团队, 中规院朱子瑜、邓东团队, 清 华大学党安荣、龙瀛团队等也都开展了这一方面的研究并取 得了丰硕成果。

目前, 第四代城市设计关于人文属性方面的研究尚有待 深人, 空间的伦理秩序、等级、行为、色彩等建成环境的认 知、识别和集体记忆的传承和新陈代谢、扬弃和建构仍然需 要完善、充实和进一步的探索。目前, 还不能明确地判断基 于数字技术发展的新一代城市设计范型能走多远，但是众多 案例的科学研究实践成果表明, 既然我们面对的是一个可以 部分通过大数据技术计量分析的城市空间形态，那么面对城 市这样一个复杂巨系统, 认知本质和趋近真理的途径就是实 存而明晰的, 最重要的是我们应该建立一个科学共同体, 并 以此去探寻基于共性科学问题解决的某种认知方法和技术解 决方案, 这种方案不仅可以通过设计成果的量化表述途径和 专利应用方式加以重复, 而且可以与先前几种范型建立结构 性关联。数字化城市设计范型的出现并非历史的偶然或者技 术变化的随机结果, 而是席卷全球的智慧城市发展潮流的必 然选择。UPI

\section{参考文献}

[1] SITTE C. Der Staedtebau nach seinen kuenstlerischen Grundsaetzen. Braunschweig: Wiesbaden, 1983

[2] CORBUSIER L. The radiant city: elements of a doctrine of urbanism to be used as the basis of our machine-age civilization[M]. Orion Press, 1967.

[3] MCHARG I L, MUMFORD L. Design with nature[M]. New York: American Museum of Natural History, 1969.

[4] 亚历山大. 新的都市设计理论 [M]. 黄瑞茂, 译. 台湾: 六合出版社, 1997 .

[5] ALEXANDER C. Notes on the synthesis of form[M]. Harvard University Press, 1964

[6]王建国.现代城市设计理论和方法 [M]. 东南大学出版社, 2011: 201.

[7] 东南大学城市规划设计研究院. 无锡城市空间形态规划研究. 2015.

[8] 东南大学城市规划设计研究院. 郑州中心城区总体城市设计. 2013.

[9] 东南大学城市规划设计研究院, 京杭运河杭州段两岸城市景观提升工 程规划. 2017.

[10] 东南大学城市规划设计研究院. 芜湖总体城市设计. 2017 .

[11] HEISENBERG W. Über den anschaulichen Inhalt der quantentheoretischen Kinematik und Mechanik[]]. Zeitschrift für Physik (in German), 1927, 43(34): 172-198.

[12] SHIRVANI H. Urban design review: a guide for planners[M]. Planners Press, 1981.

[13] BACON E N. Design of cities[M]. New York: Penguin Books, 1976.

(本文编辑: 王枫) 